\title{
LES ÉLECTIONS LÉGISLATIVES DU 20 NOVEMBRE 2011 EN ESPAGNE
}

\author{
Joan Marcet et Robert Liñeira
}

ARPos | Pôle Sud

$2012 / 1-n^{\circ} 36$
pages 127 à 139

ISSN 1262-1676

Article disponible en ligne à l'adresse:

http://www.cairn.info/revue-pole-sud-2012-1-page-127.htm

Pour citer cet article :

Marcet Joan et Liñeira Robert, « Les élections législatives du 20 novembre 2011 en Espagne »,

Pôle Sud, 2012/1 n³6, p. 127-139.

Distribution électronique Cairn.info pour ARPoS.

(C) ARPoS. Tous droits réservés pour tous pays.

La reproduction ou représentation de cet article, notamment par photocopie, n'est autorisée que dans les limites des conditions générales d'utilisation du site ou, le cas échéant, des conditions générales de la licence souscrite par votre établissement. Toute autre reproduction ou représentation, en tout ou partie, sous quelque forme et de quelque manière que ce soit, est interdite sauf accord préalable et écrit de l'éditeur, en dehors des cas prévus par la législation en vigueur en France. II est précisé que son stockage dans une base de données est également interdit. 


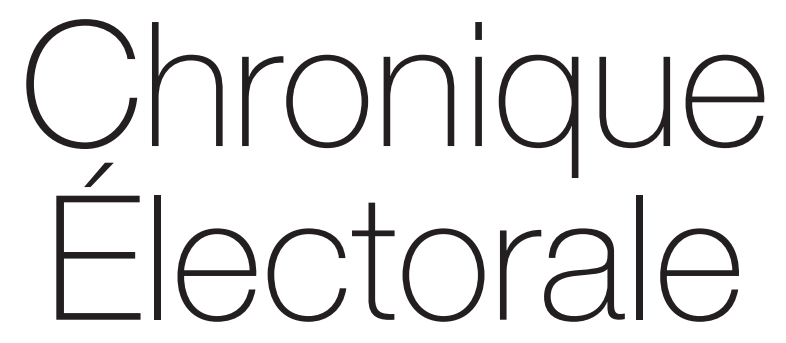




\title{
LES ÉLECTIONS LÉGISLATIVES DU 20 NOVEMBRE 2011 EN ESPAGNE
}

\author{
Joan Marcet \\ Robert Liñeira \\ Institut de Ciències Polítiques i Socials \\ (Universitat Autònoma de Barcelona) \\ Traduction : Emmanuel Négrier
}

\section{RÉsumé / Abstract}

L'ampleur de la victoire du Parti Populaire lors des élections législative du 20 novembre 2011 dans tous les secteurs et les territoires, accentue l'entrée dans un cycle long conservateur à presque tous les niveaux de gouvernement en Espagne. Le Parti populaire n'a jamais eu autant de pouvoir concentré dans ses mains. Il règne en maitre dans une grande majorité de métropoles, dans treize des dix-sept communautés autonomes et dispose d'une confortable majorité dans les deux chambres des Cortès pour gouverner l'Espagne sans le soutien d'autres forces parlementaires. Notre analyse partira d'une description de ce qui s'est passé au cours du dernier mandat du gouvernement Zapatero, pour ensuite proposer une explication du contexte politique dans lequel ces élections sont intervenues, et une interprétation des résultats. La nouvelle carte politique espagnole consacre le PP comme parti dominant la scène politique espagnole.

The magnitude of the Popular Party's vitory in legislative elections of November 2011 in all sectors and territories, underlines the deep Conservative cycle which affects almost all levels of government in Spain. The Popular Party has never had so much power concentrated in his hands. He reigns in a vast majority of cities, in thirteen of the seventeen autonomous communities and has a comfortable majority in both houses of the Parliament of Spain to govern without the support of any other parliamentary force. Our analysis starts from a description of what happened during the last term of the Zapatero government, and then proposes an explanation of the political context in which these elections have taken place, and an interpretation of such results. The new political map establishes the Spanish PP as the ruling party of the Spanish political scene.

\section{MOTS-CLÉS / KEYWORDS}

Crise économique, élections législatives, Espagne, Parti Populaire, PSOE

Economic crisis, parliamentary elections, Popular Party, PSOE, Spain 
Les élections législatives du 20 novembre 2011 en Espagne ont consacré le retour du Parti Populaire au pouvoir, après presque 8 ans de gouvernement socialiste sous la présidence de José L. Rodríguez Zapatero. La victoire du PP était annoncée par tous les sondages et enquêtes d'opinion, plusieurs mois avant l'heure, et l'on pouvait même l'anticiper au vu des résultats des élections municipales et autonomiques de mai 2011 (Marcet \& Bartomeus, 2011), qui inauguraient un nouveau cycle électoral conservateur, marqué par un fort déclin de la gauche en général et du PSOE (Parti Socialiste Ouvrier Espagnol) en particulier.

Ces nouveaux résultats ont clairement souligné ce changement de cycle. L'ampleur de la victoire du PP (Partido Popular) dans tous les secteurs et les territoires, accentue l'entrée dans un cycle long conservateur à presque tous les niveaux de gouvernement en Espagne. Le Parti populaire n'a jamais eu autant de pouvoir concentré dans ses mains. Il règne en maître dans une grande majorité de métropoles, dans treize des dix-sept communautés autonomes et dispose d'une confortable majorité dans les deux chambres du Parlement espagnol pour gouverner l'Espagne sans le soutien d'autres forces parlementaires. Les enquêtes post-électorales proposeront des interprétations approfondies du phénomène. Mais ici, notre analyse partira d'une description de ce qui s'est passé au cours du dernier mandat du gouvernement Zapatero, pour ensuite proposer une explication du contexte politique dans lequel ces élections sont intervenues, et une interprétation des résultats. La nouvelle carte politique espagnole consacre le PP comme parti dominant la scène politique espagnole.

\section{UN MANDAT MARQUÉ PAR LA CRISE ÉCONOMIQUE}

La législature qui s'achève le 20 novembre 2011 par des élections anticipées d'environ quatre mois, a été marquée, presque dès le départ, par une crise économique que tous les experts décrivent comme sans précédent. $\mathrm{Ne}$ revenons pas sur la crise qui affecte, depuis la fin 2008, l'économie occidentale en général et européenne en particulier. Mais expliquons les singularités de la crise en Espagne, qui a conduit à une nette détérioration de la confiance dans l'économie et une augmentation significative de la défiance à l'égard de la politique, et en particulier à propos de la gestion gouvernementale de la crise. C'est un même phénomène qui s'est produit dans la plupart des pays européens. En effet, en dehors des cas particuliers de la Norvège et de la Pologne, toutes les élections qui se sont déroulées depuis le début de la crise en 2008 ont été fatales aux partis au pouvoir quelle que soit leur couleur politique, et quelle que soit l'alternative politique que l'opposition proposait pour affronter la crise.

De toute évidence, comme nous le verrons, la crise n'est pas le seul facteur qui explique le changement politique du 20 novembre 2011, mais elle est un des éléments qui ont marqué le débat politique et social en Espagne, au cours des trois dernières années. Cela est dû, d'une part, aux spécificités espagnoles 
de la crise économique mondiale, et d'autre part à la façon particulière dont le gouvernement socialiste a abordé et géré la crise.

Quant au premier point, il convient de souligner : un taux de chômage en complet emballement, avec une croissance de plus de deux millions de personnes entre 2008 et 2011, et un taux très élevé pour les jeunes (avec un taux deux fois supérieur à la moyenne européenne pour les moins de 25 ans) ; une nette récession économique qui, depuis la fin de 2008, atteint tous les secteurs de l'économie espagnole, et en particulier celui de la construction qui avait agi comme un puissant levier de croissance au cours des quinze dernières années ; une augmentation persistante de la « prime de risque $\gg^{1}$, une situation largement relayée par les médias et les institutions économiques européennes. L'ensemble de ces facteurs a rendu l'acuité de la crise telle que l'indicateur de confiance économique a chuté très fortement dans les mois qui ont suivi les élections précédentes (en mars 2008). Malgré une certaine reprise et une timide sortie de la récession fin 2009, ce niveau est descendu en dessous du taux très bas de $30 \%$ dans les mois précédant les élections, comme l'indiquent les baromètres du Centro de Investigaciones Sociológicas (www.cis.es).

Si les données macroéconomiques ont été négatives pendant toute la législature, la gestion de la crise par le gouvernement Rodriguez Zapatero n'a pas été meilleure. Il passe d'une négation de la crise à un remaniement complet, en avril 2009, de l'équipe gouvernementale qui insistait sur l'urgence de mesures d'ajustement, contre l'avis du président Zapatero lui-même et son entourage ; une résistance au sommet qui cède au bout d'un an, lorsqu'en mai 2010 , le gouvernement socialiste est contraint - devant l'opinion publique et la majorité des médias ainsi que sous la pression des organismes de l'UE et de certains pays européens - de mettre en oeuvre un plan d'ajustement sévère dont l'impact sera lourd dans les secteurs sociaux sensibles à la crise (retraités, travailleurs manuels, classes moyennes et les fonctionnaires). Il entraînera d'importantes mobilisations syndicales et manifestations civiques, parmi lesquelles le mouvement qui émergea le 15 mai 2011 juste avant les élections municipales, les «Indignados ». Ce mouvement, qui a perdu en intensité au cours de l'été 2011, a néanmoins refait surface par intermittence jusqu'au mois de novembre et au-delà (Innerarity, 2011).

En fait, le mouvement du 15 mai exprime un profond malaise qui était déjà perceptible depuis plusieurs années, à partir de certains indicateurs. Ainsi, les baromètres du CIS montrent la dégradation continue de l'évaluation de la gestion gouvernementale entre avril 2008 et octobre 2011, et une perte parallèle de confiance en Zapatero, qui passe de $46 \%$, juste après sa seconde victoire de 2008, à $13 \%$ en octobre 2011 . Cette chute de confiance à l'égard de la gestion gouvernementale, que les citoyens considéraient mauvaise ou très mauvaise à $62 \%$ dans l'enquête pré-électorale du CIS, de même que la perte

1. Il s'agit de la différence en points du rendement de l'indice de référence espagnol à 10 ans par rapport à l'indice allemand. Il s'agit d'une mesure de la conjoncture économique, qui permet d'évaluer le besoin de sauver l'économie d'un pays européen. Cette différence a atteint, au cours de l'été 2011,500 points pour l'Italie et l'Espagne, un niveau considéré comme symptomatique en Grèce, en Irlande et au Portugal. 
de confiance en son président, ne correspondent pas, comme on aurait pu s'y attendre, à une plus grande crédibilité envers le principal parti d'opposition. La même enquête pré-électorale n²925 du CIS montre que $62 \%$ des citoyens estiment que le Parti populaire aurait fait la même chose ou pire s'il avait été au pouvoir. Son leader, Mariano Rajoy, est d'ailleurs aussi en net recul pendant presque toute la période, même s'il semble retrouver un certain degré de confiance juste avant les élections : elle atteint $25 \%$ en octobre 2011 , alors qu'elle était descendue jusquà $15 \%$. Différents sondages - et notamment le dernier baromètre $\mathrm{n}^{\circ} 2914$ du CIS avant les élections, qui situait cette opinion à $23 \%$ - considèrent désormais que « la classe politique » et les partis politiques sont le troisième problème du pays, après le chômage et la situation économique générale.

Depuis l'adoption de mesures d'ajustement économique en mai 2010, la gestion du gouvernement socialiste de Zapatero était conditionnée au développement de la crise dans la zone euro, et à la persistance de mauvaises indicateurs domestiques : pertes d'emplois, fermetures d'entreprises, crise et affaiblissement du secteur public. Elle était également fragilisée par la détérioration de l'image du parti au pouvoir, dont la majorité parlementaire devait, de semaine en semaine, s'appuyer sur le soutien de minorités, ce qui produisait une sensation d'instabilité et de provisoire à chaque mesure ou initiative parlementaire.

\section{LE CONTEXTE POLITIQUE DES ÉLECTIONS}

Dans un tel contexte de crise, les élections du 20 Novembre 2011 se sont tenues dans une configuration politique sans précédent. En avril 2011, un an avant la fin officielle de la législature, le premier ministre Zapatero a rendu publique sa décision de ne pas concourir à sa réélection, ce qui a renforcé l'image d'un gouvernement par intérim et a ouvert une compétition interne au sein du PSOE sur le nouveau leader. Après quelques hésitations entre candidats et à propos de la procédure (nomination, primaires internes ?) le PSOE a opté, début juillet, pour la candidat à la présidence du gouvernement d'Alfredo Perez Rubalcaba, vice-premier ministre et ministre de l'Intérieur.

À la fin juillet, le président Zapatero décidait d'avancer les élections au 20 Novembre 2011, une annonce anticipée sans précédent que l'on peut attribuer à la pression que la situation économique espagnole et européenne exerçait sur l'action gouvernementale.

Dans le contexte de confusion que cette séquence d'événements entraina dans les rangs socialistes, le Parti populaire et son candidat, Mariano Rajoy, offraient une image de force et de confiance. Rajoy avait résisté à deux défaites consécutives aux élections générales (en 2004 et 2008) et à la confrontation interne, consécutive à la défaite de 2008, au sein du congrès du Parti, en Juin de la même année (Marcet, 2010). La victoire aux élections européennes de 2009, mais surtout celle obtenue aux élections locales et régionales de mai 
2011, ont consolidé le leadership de Mariano Rajoy, et ont en partie masqué les problèmes de corruption qui impliquaient le parti, dans la Communauté Valencienne et aux Baléares.

Les autres partis politiques étaient également avides de profiter des basses eaux électorales du PSOE. À la gauche du Parti, IU (Izquiera Unida) offrait un profil radical face aux graves conséquences sociales de la crise économique, avec un nouveau candidat, Cayo Lara, chef de file de l'ancien Parti communiste, et avec des messages qui cherchaient à exploiter le mécontentement porté par le mouvement des Indignados. La nouvelle force de type nationaliste espagnol, UPD (Unión Progreso y Democracia), dirigé par une ancienne militante socialiste et qui avait obtenu déjà une siège au Congrès des Députés en 2008, ajoute à son message traditionnel une certaine dose démocrato-populiste, en tentant également de s'inspirer du mouvement du 15 mai, pour renforcer sa présence au Parlement. Quant au centre-droit nationaliste catalan $\mathrm{CiU}$ (Convergència i Unió), il se présente comme un contrepoids aux tendances centralisatrices qu'incarnent le PP et le PSOE, et comme un modèle de gestion rigoureuse de la crise. La confrontation typique de la Catalogne entre le PSC (Parti Socialiste Catalan) et CiU consacrera, pour la première fois lors d'une telle élection, la formation nationaliste catalane, malgré les coupes budgétaires dans les dépenses sociales menées depuis son retour au gouvernement catalan. Au pays basque, le débat est double : d'une part se maintient la confrontation entre le Parti nationaliste basque et le Parti socialiste, qui dirige le gouvernement régional en position minoritaire, mais avec le soutien parlementaire du PP. D'autre part se reproduit le débat politique et juridique sur les candidatures de la gauche indépendantiste, que le Tribunal Constitutionnel avait provisoirement résolu pour les élections municipales de mai 2011, avec sa décision d'autoriser les candidats de la coalition Bildu à concourir. Cette fois, il se déclare favorable à l'accès aux élections de la nouvelle coalition Amaiur, qui obtiendra un excellent résultat et deviendra la première force parlementaire basque.

Graphique 1. Cote de popularité des principaux leaders pendant la législature 2008-2011

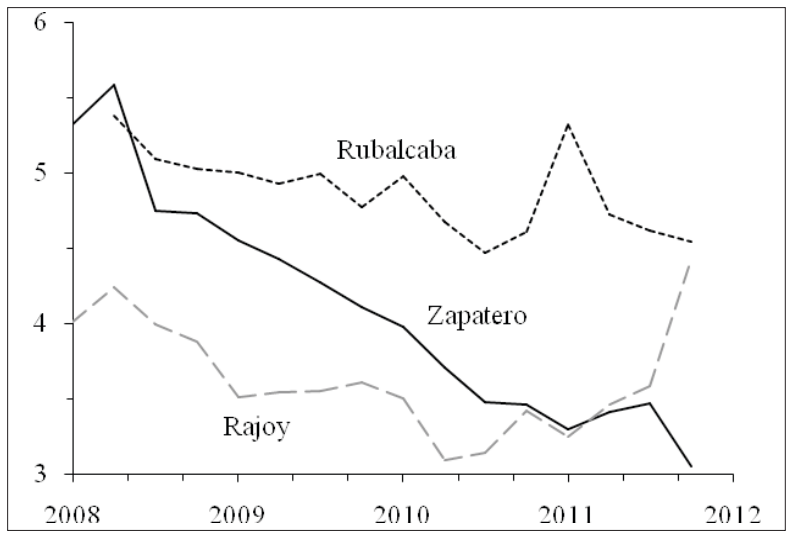


Le débat politique qui précéda les élections, entre les deux principaux partis et leaders (le PP et Mariano Rajoy, et le PSOE et son candidat d'alors, Alfredo Pérez Rubalcaba) s'est focalisé sur une confrontation entre la stratégie du PP - rentabiliser la crise économique, et présenter l'élection comme le moyen de châtier la gestion socialiste - et celle du PSOE - appuyer sur les clivages idéologiques et la distinction droite/gauche (Urquizu, 2011a). Au cours de la campagne, les enquêtes donnaient toujours le PP vainqueur avec des écarts qui oscillaient entre 12 et 16 points. Elles ne donnaient gagnant Rubalcaba que sur certains points comme le leadership, l'aptitude ou la proximité avec les gens, mais le leader PP dépassait nettement son homologue socialiste sur les thèmes majeurs de la campagne : lutte contre la crise et le chômage, confiance dans l'avenir. La campagne se résuma à un scénario attendu entre une stratégie peu dynamique de celui qui se savait déjà vainqueur, et la tentative désespérée de faire campagne et de mobiliser le vote stratégique pour le PSOE. L'annonce de l'ETA d’abandon de la lutte armée, un mois à peine avant les élections, aurait pu favoriser le candidat socialiste, ministre de l'Intérieur jusquà sa nomination comme candidat. Mais l'impact positif principal, ce fut la coalition indépendantiste de gauche basque, Amaiur, qui en bénéficia.

\section{LES RÉSULTATS : UNE INTERPRÉTATION}

Les dixièmes élections législatives espagnoles ont induit une faible participation. Seuls 69\% des électeurs se sont rendus aux urnes. En Espagne, les écarts de participation entre élections sont assez prononcés, et sont en général corrélés avec la plus ou moins grande prévisibilité du résultat. Quand le vainqueur peut être facilement anticipé, la participation se situe aux environs de 70\%. De telles élections peu compétitives ont eu lieu en 1979, 1986, 1989, $2000, \ldots$ et 2011 . En revanche, quand le résultat est incertain, la participation augmente, comme pour les législatives de 1977, 1982, 1993, 1996, 2004 et 2008 , où la participation s'est située entre $74 \%$ et $80 \%$.

En 2011, la faible mobilisation a coïncidé avec la débâcle la plus claire pour le PSOE. 29,2\% des voix et 110 sièges constituent le pire résultat du parti à une élection législative. Entre deux scrutins, les socialistes ont donc perdu en route 4 millions d'électeurs, soit le tiers de leur capital électoral (plus de 11 millions de voix en 2008). Le reflux a été généralisé sur l'ensemble du territoire, ce qui signifie que le PSOE a tout autant perdu dans ses fiefs que dans les territoires plutôt dominés par la droite. Dans toutes les communautés autonomes, le PSOE a décroché en pourcentage dans une fourchette comprise entre - $12 \%$ et $-18 \%$ des voix.

La régression a été particulièrement importante en Catalogne, non seulement en termes absolus et relatifs, mais aussi en termes symboliques. Le PSC y est passé de $45 \%$ à $27 \%$ des voix, et de 25 à 14 députés ! D'un côté, cette chute a induit que, pour la première fois dans l'histoire catalane, le PSC ne soit pas le parti vainqueur des élections, mais que ce soit $\mathrm{CiU}$, avec $29 \%$ des voix 
et 16 députés. D'un autre côté, ce recul a fait que l'avance de 17 sièges sur le $\mathrm{PP}$ en Catalogne s'est réduite à 3 sièges seulement. Cette réduction drastique d'un différentiel avec le PP qui avait joué un rôle clef dans le succès de 2008, est un autre symbole de la déroute.

Ensuite, comme il advient toujours quand le PSOE chute, les résultats furent plutôt positifs pour IU. Le parti socialiste et les ex-communistes sont très interdépendants en termes électoraux : les avancées électorales de l'un s'accompagnent de la baisse de l'autre. Ici, IU a obtenu $75 \%$ de voix en plus qu'en 2008, ce qui lui a permis de sortir de l'invisibilité parlementaire dans laquelle elle se trouvait : elle ne disposait que de deux députés sortants, et se hisse au niveau de $4^{\mathrm{ème}}$ groupe parlementaire avec 11 sièges. Ce n'est pas la seule force de gauche qui obtient de bons résultats. "Compromís ॥, une coalition du nationalisme valencian proche de IU, a même obtenu un député. La gauche du PSOE a donc tiré parti de la débâcle socialiste.

Les deux autres grands changements de ces élections sont l'essor de UPD et l'irruption de Amaiur. La première a multiplié son capital électoral par près de 3 , en obtenant $4,8 \%$ des voix et un groupe parlementaire. Avec un projet de recentralisation de l'Espagne et des éléments de rénovation du système démocratique comme bannière programmatique, UPD apparaît comme une option dont les soutiens se concentrent à Madrid, et dans les provinces centrales de l'Espagne. À l'autre bout du spectre idéologique, Amaiur, représentante du nationalisme basque, n'a pas seulement obtenu la victoire en Pays-Basque, mais a obtenu 7 députés, ce qui, sans aucun doute, la positionne comme force incontournable dans la formation de majorités lors des prochains scrutins basques, et comme acteur clef dans le processus de négociation autour de la disparition de l'ETA.

Comment tous ces mouvements sont-ils connectés ? Et, plus concrètement, où sont allés les plus de 4 millions de voix perdues par le PSOE ? Nous ne disposons pas encore de toutes les données post-électorales, mais nous pouvons nous faire une idée de ces changements, en nous attachant à leur dimension territoriale. En observant qui a gagné de l'influence électorale et qui en a perdu sur le territoire, nous pouvons nous faire une idée plus précise, même provisoire, des transferts de vote. 
Tableau 1. Résultats des élections législatives de 2011 et 2008 en Espagne

\begin{tabular}{|c|c|c|c|c|c|c|}
\hline & \multicolumn{3}{|c|}{ Élections 2011} & \multicolumn{3}{|c|}{ Élections 2008} \\
\hline & Voix & $\%$ & Sièges & Voix & $\%$ & Sièges \\
\hline PP & 10.866 .566 & 45,2 & 186 & 10.278 .010 & 40,4 & 154 \\
\hline PSOE-PSC & 7.003 .511 & 29,2 & 110 & 11.289 .335 & 44,4 & 169 \\
\hline $\mathrm{CiU}$ & 1.015 .691 & 4,2 & 16 & 779.425 & 3,1 & 10 \\
\hline IU-ICV & 1.685 .991 & 7,0 & 11 & 969.946 & 3,8 & 2 \\
\hline AMAIUR & 334.498 & 1,4 & 7 & & & \\
\hline UPyD & 1.143 .225 & 4,8 & 5 & 306.079 & 1,2 & 1 \\
\hline PNV & 324.317 & 1,4 & 5 & 306.128 & 1,2 & 6 \\
\hline ERC & 256.985 & 1,1 & 3 & 291.532 & 1,1 & 3 \\
\hline BNG & 184.037 & 0,8 & 2 & 212.543 & 0,8 & 2 \\
\hline $\mathrm{CC}$ & 143.881 & 0,6 & 2 & 174.629 & 0,7 & 2 \\
\hline COMPROMís-Q & 125.306 & 0,5 & 1 & & & \\
\hline FAC & 99.473 & 0,4 & 1 & & & \\
\hline GBAI & 42.415 & 0,2 & 1 & 62.398 & 0,2 & 1 \\
\hline Autres & 789.480 & 3,3 & & 778.659 & 3,1 & \\
\hline Blancs & 333.461 & 1,4 & & 286.182 & 1,1 & \\
\hline Nuls & 317.555 & 1,3 & & 165.576 & 0,6 & \\
\hline Abstentions & 11.113 .099 & 31,1 & & 9.172 .740 & 26,2 & \\
\hline Total & 35.779 .491 & & 350 & 35.073 .179 & & 350 \\
\hline
\end{tabular}

Source : Junta Electoral Central

Sur le graphique 2 , on retrouve la relation entre le solde électoral du PSOE et celui de ses principaux opposants politiques. Chaque point du graphique représente une des 8112 municipalités espagnoles. Sur chacune des figures se retrouvent les coordonnées cartésiennes qui délimitent les communes où le PSOE a perdu ou gagné des voix, en pourcentage sur le recensement, comparées aux pertes et gains de ses opposants sur les mêmes communes. Le dessin que présente le nuage de points décrit ainsi de façon synthétique la relation entre pertes du PSOE et gains des autres. Comme on peut le voir, le gros des points se concentre dans les troisième et quatrième quadrants, c'est-à-dire ces espaces où le PSOE a perdu son implantation. Pour le dire autrement, le PSOE a donc pratiquement partout un solde négatif vis-à-vis des autres partis et de l'abstention. 
Graphique 2. Corrélation entre les pertes du PSOE et les autres partis

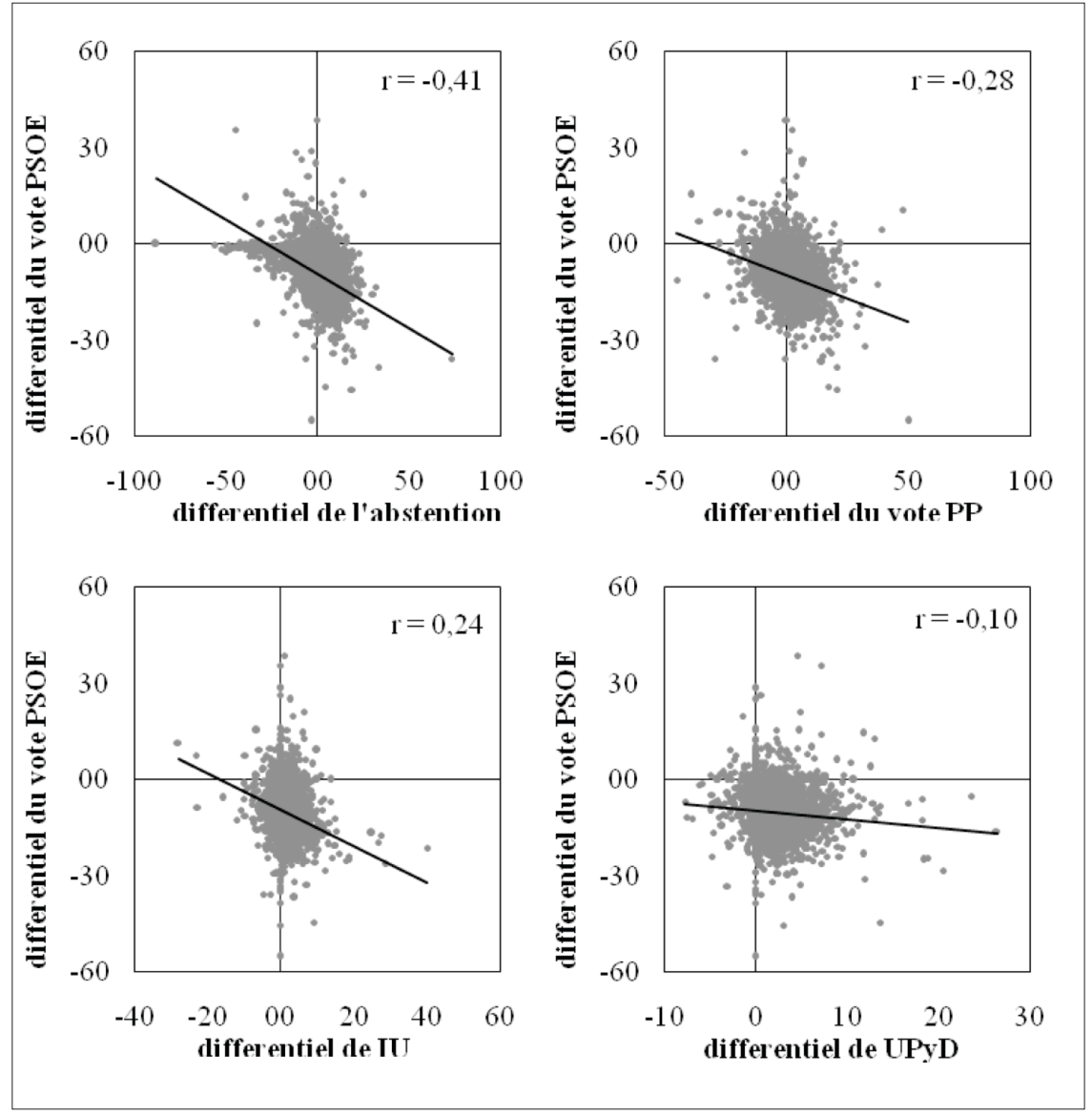

La clef d'interprétation de ces graphiques réside dans la force des associations, qui peut s'observer à travers la forme et la tendance que prend le nuage de points. Si l'on s'intéresse à la force de la relation, on observe que la plus importante des pertes du PSOE est due à l'abstention, suivie des pertes vis-àvis du PP, puis de IU. En réalité, conformément à notre estimation des pertes électorales, le taux de fidélité au PSOE (ceux qui renouvelèrent en 2011 leur confiance au PSOE de 2008) a été de 60\%. 18\% des anciens électeurs PSOE se sont abstenus. Des $20 \%$ restants, $12 \%$ environ opta pour le PP, $8 \%$ pour IU et autres partis de gauche, et $3 \%$ se dirigea vers UPD, et le reste pour diverses options2.

2. On ne doit pas oublier que cette estimation porte sur l'ensemble de l'Espagne. Dans la mesure où, surl'ensemble du territoire, plusieurs systèmes de partis existent, les mécanismes de transfert de voix peuvent fonctionner de façons distinctes selon les communautés autonomes. 
En définitive, il semble évident qu'un revers comme celui du PSOE ne peut s'expliquer par une seule cause, et n'avoir qu'un seul bénéficiaire. D'un côté, les dégâts de la crise et les valses-hésitations dans sa gestion se sont traduits par une grande incapacité à mobiliser les électeurs socialistes. Seuls $60 \%$ des électeurs socialistes de 2008 ont confirmé leur confiance au PSOE, un taux très bas comparé aux 90\% de fidélité des électeurs du PP entre 2008 et 2011. Cette faible loyauté électorale s'est traduite par l'abstention, mais aussi par la fuite vers d'autres offres politiques. On notera que la fuite vers le PP et celle vers les autres formations est d'intensité comparable. Les électeurs les plus positionnés au centre, idéologiquement, ont opté pour le PP. Il s'agit sûrement de citoyens à la recherche d'un gouvernement fort, qui s'appuient sur les préconisations de réduction du déficit dictées par l'Union Européenne, et qui ont perdu confiance dans le gouvernement au vu de sa gestion erratique de la politique économique. D'un autre côté, on trouve les socialistes plus à gauche, dont la perte de confiance à l'égard de Zapatero est sans doute due aux politiques de rigueur budgétaire déclenchées à partir de mai 2010. La crise semble donc le point focal de la défaite, même si elle a pu occasionner des transferts de voix très différents selon les segments idéologiques du parti.

\section{Conclusion}

La crise économique et sa gestion par le gouvernement Zapatero ont été sans aucun doute les thèmes majeurs de la législature et les clefs majeures d'explication de la défaite socialiste en 2011. Chronique d'une déroute annoncée, les élections ont une fois de plus montré un gouvernement européen incapable de faire face à une situation économiquement désastreuse, et châtié par les urnes. Cependant, une crise de confiance inédite à l'égard des dirigeants socialistes semble également influer de façon décisive. La crise politique, si elle atteint presque toutes les forces politiques - si l'on prend en considération la façon dont les citoyens évaluent la politique en général - a d'abord atteint le PSOE. Selon les enquêtes pré-électorales, le PSOE perdait de l'influence parmi ses électeurs les plus proches idéologiquement (Urquizu, 2011b), dans les secteurs qui l'avaient fortement soutenu lors des deux scrutins précédents. Il subissait un reflux dans le vote urbain et dans le couches les plus jeunes de la société. Au contraire, le PP montrait un renforcement, tant auprès de son électorat idéologiquement le plus proche (le vaste spectre qui va du centre-droit jusqu'à l'extrême droite), que dans les principaux centres urbains. Il dépassait également le PSOE dans les couches jeunes de l'électorat (entre 18 et 44 ans).

Même si la gravité de la crise économique fait que toute prévision de moyen terme est hasardeuse, il existe une sensation globale que les élections de 2011 marquent un changement dans la dynamique électorale espagnole. D'un côté, même si le PP dispose d'une marge qui lui permet de supporter un fort amoindrissement de sa majorité parlementaire, le PP semble disposer d'une opportunité d'étendre sa domination gouvernementale au-delà de la présente législature. La crise du PSOE, à l'inverse, est profonde. De nombreux jeunes dirigeants se sont usés au long des années de gouvernement, et 
le parti a devant lui la tâche difficile de redéfinir un nouveau projet politique, un nouveau leadership s'il veut retrouver la confiance des divers secteurs qui l'ont abandonné par la défection électorale ou la fuite vers d'autres formations politiques.

Cependant, pour construire un nouveau projet politique, le PSOE doit non seulement retrouver la centralité idéologique, un espace traditionnel de compétition qui ouvre vers la victoire électorale, mais aussi lutter contre les forces qui sont apparues sur sa gauche. Lors des élections de novembre 2011, les appels au « vote utile » n'ont pas fonctionné comme d'habitude, et le vote de gauche s'est éparpillé entre plusieurs options. Le processus de concentration du vote quavait connu le pays depuis la fin des années 1980 a connu un frein en 2011, avec l'émergence d'autres partis à la gauche du PSOE ; une gauche chaque fois plus diverse, à laquelle le PSOE doit pouvoir offrir un projet politique nouveau s'il veut à nouveau se présenter comme une alternative crédible face au PP.

En février 2012, le PSOE et le PP ont tous deux organisé leur congrès à Séville. Le premier a opté pour propulser Alfredo Pérez Rubalcaba à la tête du parti, privilégiant la solidité du vétéran, ancien ministre de Felipe González et de Rodríguez Zapatero, et candidat aux élections du 20 novembre 2011. Il a ainsi offert l'image d'unité et de cohésion interne à l'heure d'affronter une traversée du désert, durant laquelle il devra renforcer son message et son programme pour partir à l'assaut de la majorité conservatrice, au terme de 4 ans de législature. Pour sa part, le PP, encore ébloui par sa récente victoire, a renouvelé sa confiance à Mariano Rajoy qui a été capable, non seulement de reconquérir le pouvoir, mais aussi de renforcer son leadership sur le parti, en éliminant pratiquement toutes les prétentions de dissidence interne qu' il avait subies en 2008. Rajoy suscite toujours des doutes, et n'apparaît pas comme un chef de gouvernement qui aurait accru la confiance au-delà du "plus" dont bénéficient tous les nouveaux entrants, mais il dirige un gouvernement fort et mieux évalué globalement que son président lui-même, selon le baromètre du CIS n²927 de janvier 2012. L'effet des décisions gouvernementales des premiers mois, et le développement de la crise économique en Europe et en Espagne donneront une idée plus précise de sa consistance politique.

\section{Références / References}

Innerarity D., « La política después de la indignación », CLAVES de Razón Práctica, $\mathrm{n}^{\circ} 218,2011$, pp.18-30

Marcet J., Bartomeus O., «Les élections municipales et autonomiques du 22 mai en Espagne et en Catalogne », Pôle Sud, n 35, 2011, pp.129-141

Marcet J., « L'Espagne », in Reynié D. (dir.), La droite en Europe, Paris, PUF, 2012.

Urquizu I., «20-N: ¿ideología o economía? », FRC, n²7, 2011a, pp. 18-24.

Urquizu I., « Las elecciones generales de $2011 \gg$, CLAVES de Razón Práctica, no 218,2011 b, pp. 58-66. 